\title{
Review of: "Performance And Exhaust Emission Characteristics Investigation of Compression Ignition Engine Fuelled With Microalgae Biodiesel And Its Diesel Blends"
}

haitham houssein yousseif mohamed

Potential competing interests: The author(s) declared that no potential competing interests exist.

\section{Abstract,}

The results in the abstract should be declared in specific value.

Introduction;

This section was suitable, No problems

Methods;

This section needs a lot of work you should explain the Algae oil extraction method, biodiesel production method, preparation condition everything is missing in this manuscript should be clarified where are the photos of the test?

Results and discussions

This section, need a lot of work you should explain fuel properties, and what is the equation to calculate BTE, BSFC and Engine emissions.The research needs more details? 